\title{
44 Intentional weight loss
}

Weight reduction should be slow and steady because our body is not aware of our intended diet. It reacts to the food scarcity as it would react to starvation, immediately lowering the energy requirement for the organ basic functions ( $\triangleright$ Chapter 8 ). Fast weight losses are generally only transient and almost always lead to weight fluctuations that are harmful to health. According to the results produced to date by an EU-funded study in 8 countries, such yo-yo effects can be best minimized by diets with a moderately high protein content and modestly reduced glycemic index (Larsen et al. 2010).

A simple and frequently successful recommendation for committed dieters is to sensibly enjoy their food by being more conscious of what they're eating. That said, it takes approx. 30 minutes, namely, before the hormonal feedback circuitry sends the satiety signal from the stomach and intestine to the center in the hypothalamus ( $>$ Chapter 13). To avoid disappointment, an achievable weight loss goal of $5 \mathrm{~kg}$ per 150 days should be set. The ratio of body surface area to body mass over which energy is expended as heat is smaller in obese people. That is why they tend to lose weight more slowly than their lean counterparts. However, even small weight loss brings health benefits because the risk-associated abdominal fat is always broken down first and at a disproportionately faster rate.

Indeed, a low-fat meal is just as satisfying as the same amount of fat-rich fare. Such deliberations are important, seeing as when as little as only $5 \mathrm{~g}$ of the recommended carbohydrate uptake is replaced with $5 \mathrm{~g}$ of fat it leads to a weight gain of a good kilogram every year even though the amount of food eaten remains the same. Therefore, every person with weight problems is recommended to reduce their daily fat intake (Hooper et al. 2012). Fundamentally, this nutrient proportion should make up no more than $30 \%$ of the total caloric intake and should consist of $1 / 3$ saturated, $1 / 3$ monounsaturated and $1 / 3$ polyunsaturated fatty acids ( $\downarrow$ Chapter 5 and $>$ Chapter 15).

Dieting based on rigid behavioral controls or strict bans is not very helpful. Rather, it tends to lead to further eating disorders.

One concept of the presently heated debate on restricting calories and occasional fasting is that of autophagy. In this natural form of "self-digestion", the body's cells breakdown damaged or no longer required components to produce new energy equivalents or other molecules required for cellular metabolism. Thus, autophagy is not only an emergency system that kicks in during starvation phases. It is also responsible for the constant cleaning and renewal of cells (Oh et Lee 2012, Rubinsztein et al. 2012). Bacteria and viruses are similarly subject to this "spring cleaning" process. Therefore, autophagy is also a central player in our immune system (Bhattacharya et Eissa 2013). This cleaning process ensures maintenance of healthy cells and cell functions. Good recycling can therefore prolong life, but only functions on low nutritional input. That said, when we eat the 5-6 snacks and main meals we do every day, insulin is secreted constantly. However, insulin inhibits autophagy. Occasional fasting and a maximum of 2-3 meals per day in conjunction with physical activity ( $\downarrow$ Chapter 59 ), however, promote the desired effect of cellular neogenesis owing to the comparatively low insulin levels. 